\title{
Patrimonio y emociones. Estado de la cuestión desde las perspectivas internacional y nacional
}

\author{
Carlos MUNILLA GARRIDO \\ Sofía MARÍN-CEPEDA
}

Datos de contacto:

Carlos Munilla Garrido

Universidad de Valladolid

cmunilla@gmail.com

Sofía Marín-Cepeda

Universidad de Valladolid

sofiavictoria.marin@uva.es

\begin{abstract}
RESUMEN
Patrimonio y emociones son dos ejes muy relacionados desde la perspectiva humanista y relacional del patrimonio descrita en la literatura reciente (Fontal, 2013; MarínCepeda, 2018; 2018a). Estos son los dos ejes principales que guían y orientan el estudio que presentamos. Nuestro objetivo se dirige a conocer y elaborar el estado de la cuestión en torno a las relaciones entre patrimonio y emoción. Para ello, buscamos, localizamos y analizamos la presencia y relación de patrimonio y emoción en los organismos que se encargan de la gestión del patrimonio en las escalas nacional e internacional, y la literatura científica reciente. Realizamos una búsqueda a partir de descriptores específicos para cada eje y elaboramos un análisis cualitativo del estado de la cuestión en la literatura científica, los organismos internacionales (UNESCO y Consejo de Europa) y en normativas nacionales (leyes de patrimonio y planes nacionales).

Todo ello nos permite elaborar un amplio estado de la cuestión en torno a la presencia del patrimonio y las emociones en la normativa nacional e internacional y sus posibles relaciones. Para finalizar, presentamos los principales resultados y su discusión, entre los que encontramos una alta frecuencia en la presencia del patrimonio y las emociones, pero una baja incidencia en su relación e influencia en la normativa analizada.
\end{abstract}

PALABRAS CLAVE: Patrimonio; emoción; educación primaria; estado de la cuestión; formación integral. 


\title{
Heritage and emotions. State of the art from the international and national perspectives
}

\begin{abstract}
Heritage and emotions are two closely related axes from the humanistic and relational perspective of heritage described in recent literature (Fontal, 2013; Marín-Cepeda, 2018; 2018a). These are the two main axes that guide our study. Our objective is to know and elaborate the state of the art around relations between heritage and emotion. We search and analyze the presence and relationship of heritage and emotion in the management agencies of heritage at national and international scales, and recent scientific literature. We afford a search based on specific descriptors for each axis and a qualitative analysis of the state of the art in scientific literature, international organizations (UNESCO and Council of Europe) and in national regulations (heritage laws and national plans).

All of this allows us to elaborate a broad state of affairs on the presence of heritage and emotions in national and international regulations and their possible relationships. Finally, we present the main results and their discussion, among which we find a high frequency in the presence of heritage and emotions, but a low incidence in their relationship and influence in the regulations analyzed.
\end{abstract}

KEYWORDS: Heritage; emotions; primary education; state of the art; integral education.

\section{Introducción}

Esta investigación nace de la convicción del poder educativo del patrimonio como herramienta clave para la formación integral de las personas, atendiendo especialmente al cultivo de la sensibilidad, la creatividad, las emociones y el pensamiento metacognitivo.

La solidaridad, la empatía, la reflexión, la capacidad de conmovernos o de crear, entre otras, son facultades que nos convierten en entes singulares con respecto a otros seres vivos. Estas son las cualidades que realmente nos hacen humanos y son las que convierten los bienes, ya sean materiales o inmateriales, en elementos con sentido, con significado y con mensaje. Es decir, son lo que convierte lo cotidiano en singular y lo insignificante en bello. A partir de estas potencialidades, lo instrumental se transforma en cultural y, quizá, en arte y/o patrimonio.

Las características que nos convierten en una especie única son las responsables de las creaciones que, simultáneamente, nos dotan de 
humanidad. Por todo ello, las comprendemos como la mejor herramienta educativa para la formación integral con, hacia, desde y para el patrimonio.

El estudio analiza la presencia de estas variables en los principales organismos nacionales e internacionales que se encargan de gestionar el patrimonio, asi como en los documentos generados por ellos y la literatura científica circunscrita principalmente al ámbito educativo de primaria, para analizar y conocer la presencia y las relaciones entre los ejes patrimonio y emoción. Perseguimos dentro de este último objetivo, valorar iniciativas educativas que conjuguen patrimonio y emociones de forma práctica en la franja de edad 6-12 años por considerar esta etapa crucial en el desarrollo madurativo de los niños siguiendo las líneas de investigación de Bisquerra (2009, 2011, 2015).

\section{Método}

En este estudio se desarrolla un análisis del estado de la cuestión en las escalas nacional e internacional en torno al patrimonio, la emoción y sus relaciones. Para ello, acudimos a los organismos internacionales responsables de la definición y gestión del patrimonio, como son la Organización de las Naciones Unidas para la Educación, la Ciencia y la Cultura (UNESCO) y el Consejo de Europa, y a los organismos nacionales, analizando las leyes de patrimonio nacionales y autonómicas y los Planes Nacionales del Ministerio de Cultura y Deporte de España. Aplicamos una metodología basada en el análisis cualitativo a partir de la búsqueda aplicando descriptores específicos en torno a los dos ejes: patrimonio y emoción. Aplicamos para ello una metodología muy próxima a la desarrollada por Andre, Durksen y Volman (2016), quienes se apoyan en descriptores o indicadores clave para realizar un análisis comprensivo de la literatura científica.

\section{Patrimonio y emociones desde la perspectiva internacional}

El estudio del eje patrimonio y emociones comienza con el análisis de la documentación internacional de la UNESCO y el Consejo de Europa, para continuar con la escala nacional y la literatura científica.

En una primera fase, llevamos a cabo un análisis a partir de descriptores clave en torno al patrimonio en las convenciones y tratados promulgados por la UNESCO. Este análisis arroja resultados no significativos, por lo que ampliamos los descriptores de búsqueda incorporando conceptos y raíces vinculadas con lo emocional: "emoci" (emoción, emocionar, emocional, etc.), y "sentim-" (sentimiento, sentimental, etc.). A continuación, elaboramos una síntesis de los principales resultados obtenidos. 
En la Conferencia General de la UNESCO (Nueva Delhi, India), del 5 de diciembre de 1956, encontramos la Recomendación que define los Principios Internacionales que deberian aplicarse a las excavaciones arqueológicas. En el primer apéndice de este documento encontramos que la estima es uno de los elementos claves para la conservación del patrimonio. Se hace referencia a los sentimientos o emociones conectados con el valor de la educación como medio para conocer el patrimonio y aproximarlo a los pueblos fomentando su respeto (UNESCO, 1956).

La Conferencia General de la UNESCO (Paris, Francia), del 9-12 de diciembre de 1962, en su Recomendación relativa a la Protección de la Belleza y del Carácter de los Lugares y Paisajes. Se comprende la belleza como motor de lo emocional, tanto en lo contemplativo como en lo creativo, orientado hacia la conservación y restauración del patrimonio material y natural. En el documento se subraya la importancia de la belleza en torno al patrimonio, más allá del valor estético, otorgándole un papel renovador en el interior de las personas en conexión con los bienes patrimoniales en las escalas moral y espiritual (UNESCO 1962). En definitiva, se otorga al patrimonio una función crucial para la integridad de las personas y su equilibrio anímico y psíquico.

En la Conferencia General de la UNESCO (Paris, Francia), del 19 de noviembre de 1968, encontramos la Recomendación sobre la Conservación de Bienes Culturales que la ejecución de Obras Públicas o Privadas puede poner en peligro. Esta recomendación apela directamente a la vinculación emocional con los elementos patrimoniales como la medida más eficaz para la preservación de los mismos, alentando a los estados miembros de la organización a fortalecer tales sentimientos a través de la adopción de medidas adecuadas (UNESCO, 1968). Se presenta así la educación, como la vía más adecuada para la preservación del patrimonio incluyéndolo en los currículos oficiales.

Continuando con la UNESCO, en la Conferencia General (Nairobi, Kenia), de 26 de noviembre de 1976, encontramos la Recomendación relativa a la Salvaguarda de los Conjuntos Históricos o Tradicionales y su función en la vida contemporánea. Se localiza la emoción directamente relacionada con el concepto de estética. Ambas palabras son utilizadas formando un término único que, unido al concepto de armonía, busca expresar la importancia de los conjuntos históricos como generadores de sensaciones (UNESCO, 1976).

Encontramos otra referencia en la Conferencia General (Paris, Francia), de 28 de noviembre de 1978 dentro de la Declaración sobre 
los Principios Fundamentales relativos a la contribución de los Medios de Comunicación de masas, al Fortalecimiento de la Paz y la Comprensión Internacional, a la promoción de los Derechos Humanos y la lucha contra el racismo, el apartheid y la incitación a la guerra. Se trata de una referencia explícita a los sentimientos ligados a la identidad cultural dentro del apartado de cultura y comunicación.

Dentro de la Recomendación sobre la Salvaguarda y Protección de las imágenes en movimiento de la Conferencia General de la UNESCO de 1980, encontramos en uno de los principios rectores una referencia a los sentimientos ligados al arte, la identidad cultural y el patrimonio espiritual abogando por garantizar el acceso de toda la población a este bien como miembros de la comunidad humana.

Una reseña más en la Convención para la Salvaguarda del Patrimonio Inmaterial de la Conferencia General de la UNESCO en su $32^{\mathrm{a}}$ reunión (París, del 29 de septiembre al 17 de octubre de 2003). Nos resulta una referencia interesante ya que en un mismo párrafo y dentro de la misma definición encontramos una alusión a emociones y creatividad, ámbitos que pretendemos abordar de forma conjunta en otras investigaciones.

Finalmente en la Recomendación sobre paisaje urbano de la Conferencia General del año 2011 hallamos también la palabra sentimiento relacionada con la importancia de la paz para el desarrollo de la sensación de pertenencia al plano local y mundial (UNESCO, 2011).

Entendemos que la relevancia que se da a los sentimientos dentro de los más de treinta instrumentos normativos relacionados con el patrimonio y publicados por la UNESCO desde 1948 es testimonial ya que descubrimos tan solo diez alusiones. La importancia que éstas pudieran presentar de cara a su relación con lo educativo tampoco se nos antoja clave. Se liga su valor, en la mayoría de los casos, a la utilidad que pudieran presentar como medio de conservación por el fomento del afecto personal hacia los elementos patrimoniales; estimulando la identidad cultural y por tanto la pertenencia a un determinado entorno cultural común; o por medio de acciones, no concretadas en los textos, que pudieran llevar a cabo los estados miembros. De la misma manera, surge una sola vez la palabra emoción ligada a la experiencia estética, aunque exclusivamente en el entorno del patrimonio artístico. No obstante, también sorprende su escasez, máxime cuando estamos en una esfera como es la artística, donde es evidente su correspondencia en la generación y expresión de emociones.

Destacamos entre todos los hallazgos la citada referencia de 1962 
de donde extraemos un paralelismo con nuestro objetivo de búsqueda emocional. Consideramos la gestión de los sentimientos como un componente vital para un desarrollo armónico pleno de la persona a cualquier edad, pero clave en la etapa de primaria entre los seis y los doce años. Atisbamos un papel relevante al patrimonio como generador de esa buscada estabilidad personal bien sea a través de la belleza que pudiera contener, desde la identificación personal cultural como si se señala en los documentos de la UNESCO, o desde proximidad física de propiedad o adscripción con el elemento patrimonial.

Pensamos que la UNESCO considera necesario estimular los sentimientos de pertenencia de las comunidades locales primero y universales después, como mejor medio de conservación de las diferentes manifestaciones patrimoniales. Aun entendiendo que esto puede producirse de manera natural o no intencional, creemos que la educación tiene un cometido esencial en este proceso, y que recíprocamente el patrimonio también puede ser una herramienta valiosa para la educación como motor de otros aprendizajes paralelos. Es decir, es claro que se demanda que la educación ayude a la conservación del patrimonio por medio de su difusión didáctica y desde una visión moral, pero no se contempla la posibilidad de que el patrimonio pueda contribuir a educar en el más amplio sentido de la palabra.

Continuamos con el análisis de documentación aplicando los mismos descriptores al ámbito europeo. Para ello acudimos al Servicio de Publicaciones en línea EUR-Lex de la Unión Europea (UE). Esta herramienta ofrece una sintesis legislativa ordenada en treinta y dos temas relativos a todas las esferas de acción de la UE. En esta ocasión y teniendo en cuenta la temática que centra nuestra investigación nos ceñiremos a la revisión de los ámbitos de Cultura y Educación.

En el terreno dedicado a la Cultura comenzamos por el Tratado de Funcionamiento de la Unión Europea, marco de referencia para las politicas culturales y programas de cultura que se desarrollan en la actualidad. Dentro de las políticas culturales revisamos los cuatro textos existentes: las Conclusiones del Consejo de Europa sobre la promoción de la cultura y las relaciones Internacionales de la Unión Europea, el Reglamento relativo a la exportación de bienes culturales, las Conclusiones del Consejo de Europa sobre el papel de la cultura en la cooperación para el desarrollo de la Unión Europea, y la comunicación al Parlamento y al Consejo hacia una estrategia de la Unión Europea para las relaciones culturales internacionales. Ninguno de ellos presenta alusiones a emociones o sentimientos en relación con el patrimonio o la cultura. 
Las primeras referencias a las emociones las localizamos en el apartado dedicado a los programas culturales, que consta de siete iniciativas relativas a: planteamientos locales centrados en las personas, capitales europeas de la cultura, planes de trabajo culturales, innovación, programa Europa Creativa, Europeana y Sello de Patrimonio Europeo. Dentro de ellas hallamos únicamente referencias a la potenciación de sentimientos de pertenencia en torno a los espacios culturales dentro de la diversidad en tres de los documentos analizados, hasta en dos ocasiones, en la Decisión relativa a las capitales europeas de la cultura de los años 2020 al 2033. Esta misma referencia se localiza en la página 23 del Reglamento por el que se establece el Programa Europa Creativa. Lo mismo sucede con la Decisión relativa al Sello de Patrimonio Europeo, haciendo especial hincapié en los jóvenes y el desarrollo económico.

Nos centramos, a continuación, en el panorama educativo y comenzamos con el Tratado de Funcionamiento de la Unión Europea, en sus artículos 165 y 166, donde se establece el papel de la educación, la formación y la política orientada hacia los jóvenes y el deporte como elementos imprescindibles para el crecimiento, el empleo, la cualificación y cohesión de la ciudadanía.

Como vemos un resultado no significativo para nuestro objeto de estudio ya que no se toman en cuenta las emociones desde el punto de vista educativo-patrimonial en ninguno de los documentos revisados, y aunque la referencia a los sentimientos sí que la encontramos, en todas las ocasiones está referida al estímulo de los mismos sobre una cultura común europea.

Continuamos nuestro análisis documental por aquellos instrumentos legislativos relativos al ámbito educativo. Para ello en esta ocasión al no ser documentos con una temática patrimonial ni cultural en origen, cruzaremos las búsquedas relativas a emociones con las de patrimonio.

E1 análisis del documento relativo al Espacio Europeo de Educación para 2015, que localizamos en la Comunicación para reforzar la identidad europea mediante la educación y la cultura. No encontramos ninguna referencia a las emociones, pero sí algún testimonio en torno a los sentimientos ligados al espíritu de pertenencia a la UE. En éste último sentido varias referencias al patrimonio como motor para ese sentimiento de pertenencia.

En la Recomendación sobre las competencias clave en el aprendizaje permanente, dentro de la definición de conciencia y expresiones culturales, se hace alusión a las emociones señalando la utilidad de las distintas artes para expresarlas. Al hablar de 
competencias clave, encontramos menciones a sentimientos y creatividad como competencias transversales a las ocho claves. De igual forma, en este documento se hace referencia al patrimonio y la educación, no obstante no aparecen referencias conjuntas a los tres elementos: patrimonio, emociones y educación, si no agrupadas como acabamos de señalar. Finalizando el documento, en las conclusiones sobre el fomento de la creatividad y la innovación en la educación y formación, se refleja una alusión a la gestión constructiva de los sentimientos a través de las competencias clave (UNIÓN EUROPEA, 2008).

Como hemos podido apreciar los textos europeos de origen cultural presentan escasas referencias emocionales, las cuales se vinculan esencialmente a objetivos de tipo económico donde el patrimonio tiene así mismo un papel de recurso financiero.

La búsqueda de origen educativo nos reporta algún hallazgo más, y estos adquieren también algo más de importancia desde la óptica de la investigación. Vemos que se señala al patrimonio como esencial en la educación, aunque no se concreta en qué términos. En cuanto a las referencias emocionales observamos que aparecen siempre vinculadas a las competencias, bien clave o transversales, aunque también contabilizamos una alusión a las artes como medio de expresión emocional. No obstante, no localizamos referencias conjuntas a los ámbitos patrimonial, educativo y emocional.

Acudimos seguidamente a la literatura científica internacional y realizamos una búsqueda en la Web of Science (WOS) y Scopus en los idiomas inglés, francés, portugués y español, cruzando los descriptores de búsqueda patrimonio y emoción en el título de las publicaciones.

Las búsquedas en inglés arrojan un total de 5 publicaciones coincidentes que tienen en cuenta estos términos en sus títulos: “Feeling Half-Half? Exploring Relational Variation of Turkish-Heritage Young Adults' Cultural Identity Compatibility and Conflict in Austria" (Vietze, Juang, Schachner, y Werneck, 2018), "Transgressive touch: Ruination, public feeling, and the sunday times heritage Project" (Jethro, 2017), "Affect, heritage, feeling" (Crouch, 2015), "The Effects of Language Brokering Frequency and Feelings on Mexican-Heritage Youth's Mental Health and Risky Behaviors" (Kam, 2011) y "How is the national past imagined? National sentimentality, true feeling and the "heritage film", 1980-1995" (Eley, 2008).

Realizamos un análisis de sus resúmenes y encontramos que ninguno tiene un enfoque educativo, incidiendo todos ellos en los afectos y sentimientos personales o de arraigo comunitario que 
puedan despertar las diferentes manifestaciones culturales.

Al realizar la búsqueda en francés únicamente encontramos un artículo en Scopus, "Émotions patrimoniales: Quand le patrimoine devient l'affaire de tous" (Waty, 2016), que se centra en cómo el patrimonio, cuando se ve amenazado, se convierte en un foco de compromiso social para preservar y proteger la identidad.

A la vista de los resultados se amplía la búsqueda en francés a las raíces de los términos señalados, pero los únicos resultados que encontramos poseen exclusivamente enfoques psicológicos o neurocientíficos.

No obtenemos resultados en ninguno de los dos buscadores para los términos en portugués y castellano, por ello, realizamos nuevamente una búsqueda ampliada a las raíces e, incluso, extendiendo los campos de búsqueda a "todos los campos", además de la aplicación de la búsqueda avanzada con etiquetas de campo, operadores booleanos, paréntesis, etc.

\section{El patrimonio y las emociones desde la perspectiva nacional}

Analizamos en este apartado la presencia que tienen las emociones ${ }^{1}$ dentro del ámbito patrimonial en nuestro país. Para ello, revisamos la legislación vigente en materia de patrimonio, tanto nacional como autonómica. Acudimos a las iniciativas del Instituto del Patrimonio Cultural de España (IPCE) y a los Planes Nacionales del Ministerio de Cultura y Deporte, y finalizamos analizando la literatura científica reciente en nuestro país.

Atendemos a la Ley de Patrimonio Histórico y a la Ley de Patrimonio Inmaterial como referentes para el resto de leyes autonómicas. El análisis a partir de los descriptores señalados en torno a emociones y patrimonio no arroja ningún resultado.

En el análisis de la presencia de los descriptores de búsqueda en las leyes autonómicas encontramos que las comunidades de Murcia, Cataluña, Baleares, Canarias, Extremadura, Cantabria, Asturias, Castilla y León, La Rioja, Navarra, Castilla La Mancha y Madrid no hacen ningún tipo de alusión a las emociones en sus leyes patrimoniales. Tan solo 5 leyes de las 19 revisadas contienen alguna alusión. No obstante, debemos tener en cuenta que en la mayoría de ocasiones hacen referencia a la creación de un sentimiento identitario por medio de un pasado y cultura comunes basado en el patrimonio,

${ }^{1}$ Utilizaremos para la búsqueda las mismas raíces de la búsqueda internacional: "emoci” y "sentim". 
pero sin apuntar en ningún caso la utilidad de éste por medio de ese movimiento emocional interior para ayudar a los individuos a conocer o experimentar de forma consciente sus emociones.

Los Planes Nacionales se agrupan en catorce temáticas, de las que analizamos dos: el Plan Nacional de Educación y Patrimonio y el Plan Nacional de Salvaguarda del Patrimonio Cultural Inmaterial.

E1 Plan Nacional de Educación y Patrimonio es el documento, que más se aproxima a nuestra temática de investigación. En él podemos leer lo siguiente:

Tanto el conocimiento, como la comprensión o la valoración en ningún modo surgen de manera espontánea en el ser humano, sino que se adquieren a través de procesos educativos; se necesitan estrategias adecuadas que nos ayuden a conocer el Patrimonio, ya sea a partir de los sentidos, desde la emoción, desde el pensamiento o desde la propia actuación. Por lo mismo, las estrategias didácticas nos ayudan a comprender, nos animan a buscar el sentido, a otorgar los valores mediante procesos de investigación, experimentación, deducción, reflexión, etc. (Plan Nacional de Educación y Patrimonio, 2015, p. 17).

Resulta apropiado abordar más en profundidad las estrategias para conocer el patrimonio a las que alude el Plan Nacional:

- A través de los sentidos: por medio de iniciativas de investigación-acción podemos acercarnos al patrimonio desde sentidos, como el gusto, o bien utilizando tecnologías digitales, tal y como queda recogido en las iniciativas del Proyecto IGNIS 2 .

- Desde la emoción: el interés radica en el uso de aquello que es capaz de despertar dentro de cada observador. Podemos encontrar nuevamente un ejemplo práctico en IGNIS ${ }^{3}$, asociando los colores a las emociones básicas, expresándolas por medio de onomatopeyas, sonidos cotidianos o sonidos de instrumentos musicales.

- Desde el pensamiento: la publicación de Munilla (2017), "Patrimonio y creatividad: miradas educativas", aborda esta visión, definiéndola como "Heritage Thinking".

- Desde la propia actuación: es importante el acceso al patrimonio vivenciándolo en primera persona, independientemente de disponer de mediadores o iniciativas de otro tipo que faciliten el acceso al mismo.

\footnotetext{
2 https://proyectoignis.es/investigacion/sentidos/

3 https://proyectoignis.es/investigacion/emociones-colores-y-sonidos/
} 
Encontramos una única cita emocional relevante para este análisis dentro del apartado de innovación, donde se describen los objetivos que garantizan la calidad de las líneas de actuación del plan. Se señala la necesidad de potenciar los aspectos emocionales y sensoriales del patrimonio, subrayando su valor simbólico e identitario (Plan Nacional de Educación y Patrimonio, 2015. p.28).

Nuevamente, como se evidencia, en la relación emoción-patrimonio la primera es relegada a una utilidad de potenciación identitaria. No obstante, en ésta última cita la referencia sensorial entendemos que deja abierta la puerta a una utilización del patrimonio en este sentido, y nos hace albergar esperanzas de una posible expansión del uso de elementos patrimoniales a los que se accede por todos los sentidos. De esta forma encontramos un apoyo a la propuesta ya referida de proyecto IGNIS y el enfoque de este estudio. En cualquier caso y teniendo en cuenta que aludimos a un documento educativo no podemos por menos que señalar que la escasez de referencias emocionales es muy significativa con respecto al papel que se le otorga.

En el Plan Nacional para la Salvaguarda del Patrimonio Cultural Inmaterial, se describen las características del Patrimonio Cultural Inmaterial (PCI), considerándolo parte de la identidad de individuos y comunidades, ya que está interiorizado en ellos y eso lo diferencia de otros patrimonios (Plan Nacional para la Salvaguarda del Patrimonio Cultural Inmaterial, 2015). Destacamos de las aportaciones de este plan dos elementos: por un lado, el espacial ya que numerosas actividades y celebraciones tienen un lugar concreto de referencia que, en ocasiones, le otorga un significado especial; y por otro, la importancia de lo material como soporte de significado e información, a lo que se denomina cultura inmaterial. Ambas se consideran importantes como medio de refuerzo identitario y como vía de conservación del PCI junto con la investigación, siempre en relación al sentimiento de apego hacia el PCI. (Plan Nacional para la Salvaguarda del Patrimonio Cultural Inmaterial, 2015).

Como se evidencia en esta revisión en torno a las emociones y sentimientos, el PCI es el que más conexión presenta con este tipo de elementos personales individuales o colectivos que están muy enraizados en la sociedad, por medio de fiestas y tradiciones que, en ocasiones, se consideran como la mejor representación de un colectivo. No obstante, insistimos nuevamente en la ausencia de referencias que conjuguen en algún momento las reseñas emocionales con el ámbito educativo más allá del evidente, y repetidas veces aludido, valor identitario. 


\section{Análisis de la literatura científica en torno al patrimonio y las emociones}

En la revisión de las bases de datos Web of Science (WOS) y Scopus realizada en el análisis internacional, ya se analizaron artículos en castellano por lo que en esta ocasión vamos a complementarlas con búsquedas en el portal bibliográfico Dialnet, la base de datos de tesis doctorales Teseo y el buscador especializado Google Académico atendiendo en la búsqueda a trabajos fin de grado y trabajos fin de master. Para ello, utilizamos como descriptores de búsqueda patrimonio y emociones, combinados en el campo de búsqueda relativo al "título" de las publicaciones educativas circunscritas a la educación primaria. No obstante, y por su cercanía con nuestro objeto de estudio, aunque dentro de la etapa de secundaria, citamos los estudios de Trabajo y Cuenca (2017), y Fontal (2007). En esta misma línea, pero dentro de la etapa de infantil reseñamos el trabajo de Rusillo (2017), a la par que el de Huerta y Franco (2011) centrado en el alumnado de magisterio.

Entre los autores localizados con incidencia directa dentro del estudio por sus implicaciones en la etapa de primaria cabe destacar a Santacana (2014) y en especial el artículo "El Patrimonio Cultural y el Sistema Emocional: un estado de la cuestión desde la didáctica" (Santacana y Martínez, 2018), donde se analiza la relación entre factores emocionales y patrimonio. Los autores afirman que el patrimonio es una herramienta educativa que contiene un alto potencial de emotividad. Por nuestra parte posicionamos al lado de los autores, tal y como recogemos en anteriores publicaciones, subrayando su potencialidad como herramienta para la formación integral de la persona (Munilla, 2014; 2018).

Continuando con Santacana, localizamos referencias de interés en su libro "El patrimonio cultural inmaterial y su didáctica” (Santacana, Llonch y Martínez, 2015). Donde se presenta un enfoque motivador de las emociones: "aquí hay una relación biyectiva mediante la cual las emociones estimulan la motivación y la motivación desencadena emociones; son dos caras de la misma moneda." (Santacana, Llonch y Martinez, 2015, p.107) resulta interesante por su cercanía a nuestras propuestas la oferta de utilización de las emociones como herramienta para conseguir otros objetivos educativos.

Conviene destacar el trabajo emocional basado en objetos concretos como motores desencadenantes de emociones (Santacana, 2017). Entendemos que cuando Santacana habla de objetos "procedentes del pasado" engloba en este término objetos arqueológicos, artísticos, instrumentales, arquitectónicos, etc., es 
decir, cualquier objeto susceptible de evocar emociones, por lo tanto, hablamos de cualquier elemento material, todo aquello que desde su naturaleza tangible porta las cargas humanas de creadores, usuarios, admiradores, detractores, etc.

Continuando con nuestro análisis, destacan las aportaciones de López Facal desde la Universidad de Santiago de Compostela. El autor diserta sobre los usos del patrimonio para la instrumentalización política y la necesidad de despertar la mirada crítica de la ciudadanía sobre ello. Afirma que lo patrimonial, cuando tiene un interés de uniformización en la identidad de la sociedad, es de gran poder porque, inmediatamente después de la valorización patrimonial, está la identificación emocional (Castro y López Facal, 2017).

Son de gran interés otras de sus publicaciones, como "La comprensión del paisaje partiendo de las emociones", donde desarrolla un estudio de caso donde aborda el uso de las emociones, el trabajo cooperativo o los contenidos multidisciplinares como modelo de integración y compromiso social (Rivero, López Facal, y López, 2017).

Conviene citar, así mismo, las publicaciones del grupo de investigación "Educación Patrimonial para la Inteligencia Territorial y Emocional de la Ciudadanía" (EPITEC) de la Universidad de Huelva. En su publicación "Educación Patrimonial e Inteligencia Emocional. Hablan los alumnos" se afirma que el alumnado comprende el patrimonio como medio para despertar en ellos actitudes como el entusiasmo o el compromiso (Lucas y Estepa, 2017, p.204). En otra de sus publicaciones, se afirma que:

La educación emocional puede ayudarnos a trabajar el desarrollo global del alumnado promoviendo la construcción de ciudadanos capaces de convivir en nuestra sociedad respetando los derechos humanos y las libertades fundamentales, a través del conocimiento de las emociones y la regulación de las mismas. (Gómez y Cuenca, 2017, p.251).

El artículo describe una propuesta metodológica de trabajo para infantil o primaria que analiza estrategias y recursos didácticos que ayuden a desarrollar las emociones a través de la Educación Patrimonial.

Encontramos varias referencias en Álvarez Domínguez, quien propone lo emocional en conexión con los museos pedagógicos. El autor afirma que cualquier tipo de museo tiene un objetivo de provocación emocional. El museo debe atender a la formación integral de la persona y no solamente al aprendizaje (Álvarez, 2007). Además de 
insistir en la aportación que los museos pueden hacer a la educación emocional, añade la transmisión de valores éticos (Álvarez, 2013).

Ansorena conecta la emoción y el patrimonio, en este caso inmaterial, a través de la narración de la iniciativa de un archivo oral navarro. El autor relaciona el valor del patrimonio inmaterial y la educación emocional como elementos esenciales para formar ciudadanos (Ansorena, 2005).

Desde la Universidad Pública de Navarra y en relación al patrimonio hablado y su conexión con las emociones como la alegría, la tristeza y el miedo y su conceptualización en el lenguaje castellano y euskera, localizamos una publicación de Aznarez y Santazilia (2016).

Para terminar, destacamos la idea de la emoción como eje para la comprensión de la utilidad de determinados elementos patrimoniales y su poder educativo integral e integrador (Arias, 2017).

En el buscador de tesis doctorales "Teseo" no localizamos ninguna referencia a patrimonio y emociones en los títulos. Encontramos hasta 106 referencias si realizamos el filtrado en el resumen, pero al revisar estos resultados no nos resultan válidos puesto que presentan enfoques sin relación con la educación primaria, de tipo turístico, publicitario, arquitectónico, etc. Este mismo problema se nos presenta en los resultados de Dialnet y Google Académico. No obstante ampliamos la búsqueda a trabajos fin de grado y fin de máster donde encontramos algunos resultados.

Es el caso de Zorzo y Arche (2018) que nos narran la visión del proyecto IDEAS un modelo de centro que tiene como eje las emociones y el patrimonio natural y socia-cultural. O el de Morente (2017) donde aparecen algunas referencias emocionales basadas en el concepto inteligencia emocional de Goleman (2001) y las inteligencias múltiples de Gardner (2011) desde las que se propone una unidad didáctica de acceso al patrimonio histórico monumental.

Para finalizar este apartado, nos atrevemos a afirmar que el patrimonio es tenido muy en cuenta en la literatura analizada desde el punto de vista educativo y que, como hemos visto, se relaciona frecuentemente con las emociones de forma transversal como elemento de valor formativo e instruccional. No obstante, no localizamos un número significativo de experiencias educativas prácticas donde estos aspectos sean eje central educativo o, al menos, cuerpos teóricos que guíen el diseño de las prácticas educativas hacia una mejor y mayor conexión entre patrimonio y emoción. 


\section{Discusión}

A la luz de los resultados obtenidos en los diferentes ámbitos analizados, podemos afirmar que patrimonio y emociones tienen presencia en los documentos considerados tanto en la escala internacional como nacional. Entendemos que están presentes, pero, al igual que sucede en la literatura científica estudiada, no se presentan relacionados ni con el peso suficiente para funcionar como ejes vertebradores de planes específicos.

Vemos que la UNESCO considera relevante el patrimonio como elemento de cohesión social y de pertenencia grupal. Una vez conseguido este fin entiende que esto puede ayudar a la conservación del propio patrimonio.

Por otro lado, la documentación europea apela a la sensibilización patrimonial como elemento para crear una conciencia de cultura común, utilidad que también recalcan los planes nacionales. Finalmente, las leyes autonómicas centran sus alusiones en la creación de un sentimiento identitario.

Advertimos, por tanto, que la documentación de organismos oficiales concentra sus intereses en la utilización del patrimonio como herramienta para estimular sentimientos de pertenencia restringidos a los territorios que abarca cada entidad, mundial, continental, nacional o autonómica.

En cuanto a la literatura científica, vemos que pese a enfocar los elementos de búsqueda de manera menos utilitaria y relacionarlos más directamente con su valor educativo, no utiliza ambos a la vez de manera indivisible.

Entendemos, por tanto, que en casi ninguno de los niveles analizados se entiende que el patrimonio y las emociones puedan tener un valor educativo con una utilización conjunta de los mismos, con alguna excepción en las propuestas didácticas. Vemos que se les otorga cierta importancia de manera individual, pero con otros objetivos distintos a los propuestos en nuestro enfoque. Las alusiones que parten del ámbito educativo son muy generales y sin apenas concreción práctica, por lo que es dificil que puedan servir de germen para el nacimiento de iniciativas educativo-patrimonio-emocionales con cabida en el currículum de primaria.

Creemos, por ello, que existe un campo interesante por desarrollar. Vemos que la importancia dada a cada uno de los tres elementos por separado, otorgándoles aptitudes esenciales en la formación de la personalidad y la incorporación a una identidad cultural, puede multiplicarse exponencialmente con una propuesta de trabajo conjunta tanto en su consideración educativa como en su valor pedagógico de formación integral.

\section{Referencias}

Álvarez, P. (2007). Educación emocional, desarrollo de la afectividad y museos pedagógicos. En A. Bernal-Guerrero, Afectividad y educación en la sociedad globalizada: actas de las IV Jornadas Pedagógicas de la 
Persona, pp. 14-36. Sevilla: Universidad de Sevilla.

Álvarez, P. (2013). Educar en emociones y transmitir valores éticos: un desafio para los Museos de Pedagogía, Enseñanza y Educación. Educació i Història: Revista d'Història de l'Educació, 22, pp. 93-116. doi: 10.2436/20.3009.01.118.

Andre, L., Durksen, T. y Volman, M.L. (2016). Museums as avenues of learning for children: a decade of research. Learning Environments Research, 20(1), pp. 47-76.

Arias, N. (2017). Patrimonio y emociones: educando para una sociedad integradora. En R. De la Fuente y C. Munilla (Eds.), Patrimonio y creatividad miradas educativass, pp 309-319. Valladolid: Verdelis.

Ansorena, A. A. (2005). Archivo del patrimonio oral e inmaterial navarro: polifonía de emociones. Cuadernos de etnología y etnografia de Navarra, 37(80), 7-22.

Aznárez, M., \& Santazilia, E. (2016). Un acercamiento a la conceptualización de algunas emociones en el patrimonio fraseológico del euskera y del castellano. Huarte de San Juan. Filología y Didáctica de la Lengua, 13-33.

Castro, B. y López Facal, R. (2017). De patrimonio nacional a patrimonio emocional. HER\&MUS monografias, 18, pp. 41-53.

Crouch, D. (2015). Affect, heritage, feeling. The Palgrave Handbook of Contemporary Heritage Research. pp. 177-190.

Bisquerra, R. (2009). Psicopedagogía de las emociones. Madrid. Síntesis.

Bisquerra, R. (2011). Educación Emocional. Propuestas para educadores y familias. Bilbao. Descleé de Brouwer.

Bisquerra, R. (2015). Inteligencia emocional en educación. Madrid. Sintesis.

Eley, G. (2008). How is the national past imagined? National sentimentality, true feeling and the "heritage film", 1980-1995. Gender, Labour, War and Empire: Essays on Modern Britain. pp. 238-255.

Fontal, O. y Marín-Cepeda, S. (2018). El Observatorio de Educación Patrimonial. Revisión de experiencias y estudios sobre la base del enfoque relacional. En M. Alcántara, M. García y F. Sánchez (Coords.), Arte y Patrimonio Cultural: 56 Congreso Internacional de Americanistas, 3.

(2018a). Nudos Patrimoniales. Análisis de los vínculos de las personas con el patrimonio personal. Arte, Individuo y Sociedad 30 (3), 483500 .

Fontal, O. (2007). El Patrimonio cultural del entorno próximo, un diseño de sensibilización para secundaria. Enseñanza de las Ciencias Sociales. 
Investigación didáctica, 6 , ps.š̉ß 1-47.

Gardner, H. (2011). Las inteligencias múltiples: la teoría en la práctica. Barcelona. Paidós Ibérica.

Goleman, D. (2001). Inteligencia Emocional. Barcelona. Kairós.

Gómez, I. y Cuenca J. M. (2017). Trabajar las emociones desde la educación patrimonial para atender la diversidad del aula. En R. Martínez, R. García-Morís y C. R. García, Investigación en didáctica de las Ciencias Sociales. Retos, preguntas y líneas de investigación, pp. 649-657. Córdoba: Universidad de Córdoba.

Huerta, R. y Franco, C. (2011) Público y privado. Espacios urbanos observados por el alumnado de magisterio. Íber. Didáctica de las Ciencias Sociales, Geografia e eis

Instituto del Patrimonio Cultural de España (2015). Plan Nacional de Educación y Patrimonio. Madrid: IPCE.

Instituto del Patrimonio Cultural de España (2015). Plan Nacional para la Salvaguarda del Patrimonio Cultural Inmaterial. Madrid: IPCE.

Rusillo, M. (2017). El patrimonio cultural despiertașẹphuestras emociones: un proyecto educativo para la educación infantil. Trabajo Fin de Grado. Universidad de Granada

Jethro, D. (2017). Transgressive touch: Ruination, public feeling, and the sunday times heritage Project. Public art in South Africa: Bronze warriors and plastic presidents. pp.143-165.

Kam, J.A. (2011). The Effects of Language Brokering Frequency and Feelings on Mexican-Heritage Youth's Mental Health and Risky Behaviors. Journal of Communication

61(3), pp. 455-475.

Lucas, L y Estepa, J. (2017). Educación Patrimonial e Inteligencia Emocional. Hablan los alumnos. CLIO. History and History teaching, 41. Recuperado de: http://clio.rediris.es

Munilla, C. (2014). Educación Patrimonial versus Educación Integral. Polos opuestos o almas gemelas. Segovia Histórica, 1, pp. 287-294.

Munilla, C. (2017). Prólogo. Heritage Thinking. En R. de la Fuente y C. Munilla, Patrimonio y Creatividad Miradas Educativas, pp. 7-9. Valladolid: Verdelis.

Munilla, C. (2018). Prólogo. En R. de la Fuente y C. Munilla, Visiones Transdisciplinares en torno a Patrimonio, Creatividad y Poesía, pp. 78. Valladolid: Verdelis.

Rivero, T., López, M. y López Facal, R. (2017). La compresión del paisaje partiendo de las emociones. En R. Martínez, R. Garcia-Morís y C. R. García, Investigación en didáctica de las Ciencias Sociales. Retos, preguntas y lineas de investigación, pp. 751-761. Córdoba: 


\section{Universidad de Córdoba.}

Santacana, J. (2014). El patrimonio, la educación y el factor emocional. En II Seminário Internacional de Educação Patrimonial: contributos para a construção de uma consciência patrimonial, Braga, 4-5 Diciembre 2014. Recuperado de: https:/ / didcticadelpatrimonicultural.blogspot.com/2014/11/elpatrimonio-la-educacion-y-el-factor.html

Santacana, J. (2017). Reflexión sobre el patrimonio cultural, la educación y las identidades. RUNAE, (1), pp. 67-82. Recuperado de: http://runae.info/index.php/RUNAE/article/view/9

Santacana, J. y Martínez, T. (2018). El patrimonio cultural y el sistema emocional: un estado de la cuestión desde la didáctica. Arbor, 194 (788), pp. 446. Recuperado de: https://doi.org/10.3989/arbor.2018.788n2006

Santacana, J., Llonch, N. y Martínez, T. (2015). Las emociones y el patrimonio inmaterial. En J. Santacana y N. Llonch (Eds.), El patrimonio cultural inmaterial y su didáctica, pp. 104-108. Gijón: Trea.

Trabajo, M. y Cuenca, J.M . (2017). La educación patrimonial para la adquisición de competencias emocionales y territoriales del alumnado de enseñanza secundaria. Pulso: revista de educación, N40, pp. 159174.

Recuperado

de: https://revistas.cardenalcisneros.es/index.php/PULSO/article/view/ $\underline{261}$

UNESCO. Recomendación que define los Principios Internacionales que deberian aplicarse a las Excavaciones Arqueológicas. Conferencia General de la Organización de las Naciones Unidas para la Educación, la Ciencia y la Cultura, Nueva Delhi (India) 5 de diciembre de 1956.

UNESCO. Recomendación relativa a la protección de la Belleza y el Carácter de los Lugares y Paisajes. Conferencia General de la Organización de las Naciones Unidas para la Educación, la Ciencia y la Cultura, Paris (Francia) 9-12 de diciembre de 1962.

UNESCO. Recomendación sobre la Conservación de los Bienes Culturales que la Ejecución de Obras Públicas o privadas pueda poner en peligro. Conferencia General de la Organización de las Naciones Unidas para la Educación, la Ciencia y la Cultura, Paris (Francia) 19 de noviembre de 1968.

UNESCO. Recomendación relativa a la Salvaguarda de los Conjuntos Históricos o Tradicionales y su Función en la vida Contemporánea. Conferencia General de la Organización de las Naciones Unidas para la Educación, la Ciencia y la Cultura, Nairobi (Kenia) 26 de octubre30 de noviembre de 1976. 
UNESCO. Convención para la salvaguarda del patrimonio cultural inmaterial. Conferencia General de la Organización de las Naciones Unidas para la Educación, la Ciencia y la Cultura. París (Francia) 29 de septiembre- 17 de octubre de 2003.

UNESCO. Recomendación sobre el paisaje urbano histórico. Conferencia General de la Organización de las Naciones Unidas para la Educación, la Ciencia y la Cultura. París (Francia) 25 de octubre- 10 de noviembre de 2011.

UNIÓN EUROPEA. Decisión n. ${ }^{\circ}$ 1622/2006/CE del Parlamento Europeo y del Consejo, de 24 de octubre de 2006, por la que se establece una acción comunitaria en favor de la manifestación "Capital Europea de la Cultural" para los años 2007 a 2019 (DO L 304 de 3.11.2006, pp. 1-6). Diario oficial de la Unión Europea.

UNIÓN EUROPEA. Recomendación 2006/962/CE del Parlamento Europeo y del Consejo, de 18 de diciembre de 2006, sobre las competencias clave para el aprendizaje permanente (DO L 394 de 30.12.2006, pp. 10-18). Diario oficial de la Unión Europea.

UNIÓN EUROPEA. Versión consolidada del Tratado de Funcionamiento de la Unión Europea. Diario oficial de la Unión Europea. Tratado de Lisboa 13 de diciembre de 2007. C 202 de 7.6.2016.

UNIÓN EUROPEA. Conclusiones del Consejo y de los Representantes de los Estados miembros, reunidos en el seno del Consejo, de 22 de mayo de 2008, sobre el fomento de la creatividad y la innovación en la educación y la formación. Diario oficial de la Unión Europea.

UNIÓN EUROPEA. Decisión no 1194/2011/UE del Parlamento Europeo y del Consejo, de 16 de noviembre de 2011, por la que se establece una acción de la Unión Europea relativa al Sello de Patrimonio Europeo (DO L 303 de 22.11.2011, pp. 1-9). Diario oficial de la Unión Europea.

UNIÓN EUROPEA. Reglamento (UE) no1295/2013 del Parlamento Europeo y del Consejo, de 11 de diciembre de 2012, por el que se establece el Programa Europa Creativa (2014 a 2020). Diario oficial de la Unión Europea.

UNIÓN EUROPEA. Comunicación de la Comisión al Parlamento Europeo, al Consejo, al Comité Económico y Social Europeo y al Comité de las Regiones: Reforzar la identidad europea mediante la Educación y la Cultura. La contribución de la Comisión Europea a la reunión de dirigentes en Gotemburgo, 17 de noviembre de 2017 [COM(2017) 673 final, 14.11.2017]

Vietze, J., Juang, L., Schachner, M.K., Werneck, H. (2018). Feeling HalfHalf? Exploring Relational Variation of Turkish-Heritage Young Adults' Cultural Identity Compatibility and Conflict in Austria. Identity, 18:1, pp. 60-76, DOI: 10.1080/15283488.2017.1410159. 
Waty, B. (2016). Émotions patrimoniales: Quand le patrimoine devient l'affaire de tous. Bulletin des Biblioteques de France, (7), pp. 9-22.

Zorzo, A y Arche, V. (2018). Área educativa para la emoción como vehículo del aprendizaje de patrimonio natural y socio-cultural: Proyecto "Ideas. (TFM). Recuperado de: https: / /ibros.uam.es $/$ ?press $=$ tfm\&page $=$ catalog\&op=book\&path $\% 5 \mathrm{~B}$ \%5D=706 